\section{Moving 3D Cell Cultures from Bench to Practice: TEDD Annual Meeting at ZHAW Waedenswil 22 October 2015}

\section{Elsbeth Heinzelmann, Journalist science + technology}

Abstract: Three-dimensional (3D) cell culture technology captures the market! Driving forces include an increase in cancer worldwide, but also reinforced research for biopharmaceutical products to cure diseases and to reduce animal experiments. The challenge now is to translate in vitro assays into relevant in vivo outcomes. At the TEDD Annual Meeting, selected experts explored this problem and discussed how to move 3D cell cultures from bench to practice.

Keywords: 3D cell cultures - Tissue Engineering for Drug Development and Substance Testing (TEDD)

The BCC Research Biotechnology Report sends a clear message: the 3D cell culture market was valued at US\$ 438.1 million in 2013 and is expected to grow to about US \$ 2.2 billion in 2019. The technology has found its way into drug discovery and development. "Today the challenge is to translate in vitro assays into routinely used products", states Prof. Ursula Graf-Hausner, initiator of the TEDD network and organizer of the conference. "3D cell culture enters its next development phase with technology maturation, optimization of assays, protocols and kits and prevalidation. The goal is to implement easy-to-handle biomimetic tissue constructs that simulate the in vivo condition in a reproducible and reliable way." The TEDD network (Tissue Engineering for Drug Development and Substance Testing) wanted to know exactly what was going on and on October 22, 2015 - the day of its $5^{\text {th }}$ anniversary - invited internationally recognized experts from academia and industry to present the latest innovations.

\section{Cell-instructive Polymer Matric Platforms}

For Carsten Werner, director of the Institute of Biofunctional Polymer Materials at the Leibniz Institute of Polymer Research Dresden and professor at the TU Dresden, the modulation of cellinstructive signals of extracellular matrices (ECM) in robust and thoroughly defined ways is a key challenge in tissue engineering and regenerative medicine. Reconstituted ECM assemblies and defined, ECM-inspired polymer structures can be instrumental in deciphering molecular mechanisms of tissue formation, homeostasis and wound healing. Hence the recent attempts to explore several dedicated polymer matrix platforms combining the defined provision of adhesion receptor ligands, physical cues, the presentation of morphogens and tailored degradability. With his group, Carsten Werner has developed protocols for the reliable anchorage of native cell-secreted ECM to culture carriers. The approach was validated in the culture of human mesenchymal (MSC) and hematopoietic stem and progenitor cells (HSPC) in vitro, demonstrating the unique ability of the cell-secreted matrices to modulate expansion and differentiation of the bone marrow stem cells. The researchers also introduced a rational design strategy for ECM-inspired hydrogel networks consisting of multi-armed polyethylene glycol, sulfated glycosaminoglycans and functional peptide units to allow for the decoupling of biochemical and mechanical matrix properties. Using this approach, the scientist identified different combinations of matrix characteristics to effectively stimulate therapeutically relevant cell fate decisions.

http://www.ipfdd.de/en/departments/institute-of-biofunctional-polymer-materials/

\section{Results from Frontline Research}

In short talks, scientists in the TEDD network provided insights into the current status of science and research in their laboratories.

Dr. Cornelia Lee-Thedieck is Junior Group Leader of Stem Cell-Material Interactions at the Karlsruhe Institute of Technology. In her talk she focused on the Potential of Macroporous Hydrogels as Biomimetic 3D Bone Marrow Analogs.

Hematopoietic stem cells (HSC) replenish the blood system with all types of blood cells, including red blood cells and immune cells. The HSC niche is a complex, 3D environment in which HSCs are in contact with other cells and the extracellular matrix. They are influenced by biological signals such as cellcell-contacts as well as biophysical cues, including substrate nanostructure and elasticity, which are not reflected by standard cell culture in 2D tissue culture plates. Therefore, Cornelia LeeThedieck and her colleagues design biomaterials to study the in-

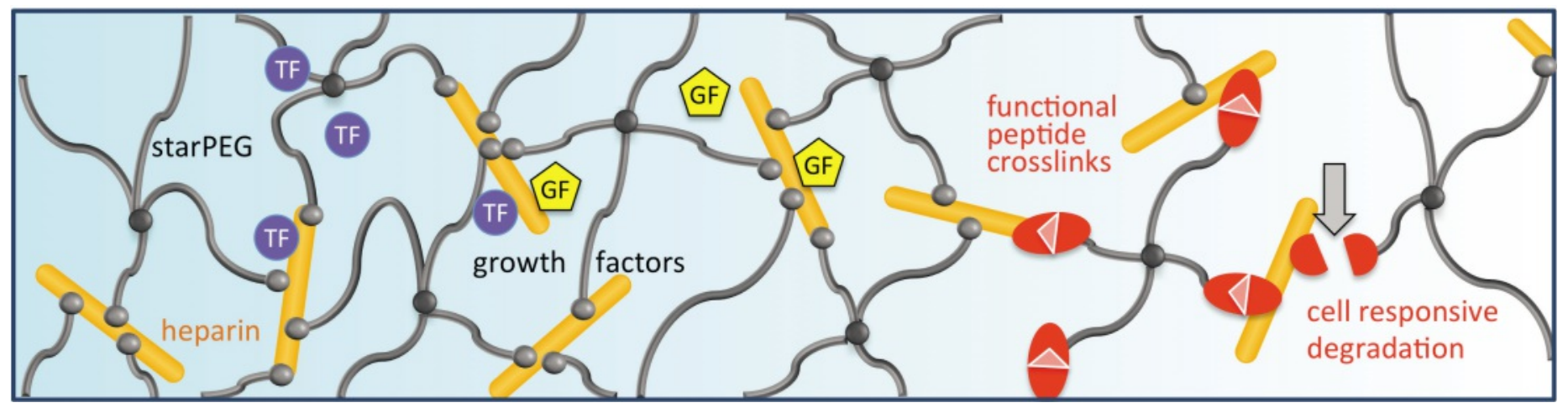

Heparin-starPEG gel platform decoupled gradation of physical and biomolecular signals. Figure: IPFDD Dresden. 


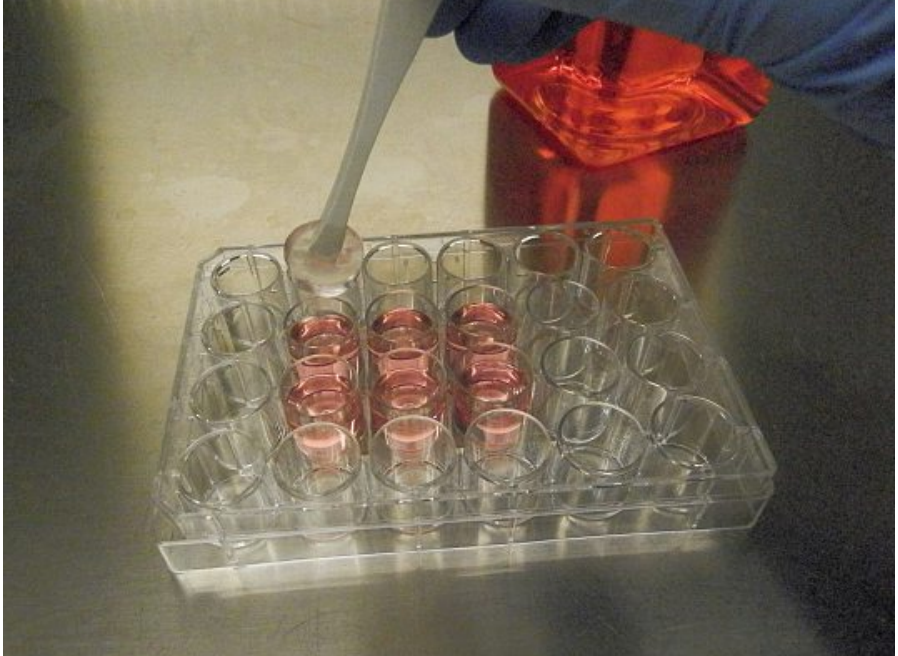

A macroporous 3D hydrogel resembling trabecular bone which, in nature, accommodates the red bone marrow with HSC niches, is placed into a vessel for the cultivation of stem cells. Photo: C. LeeThedieck/KIT.

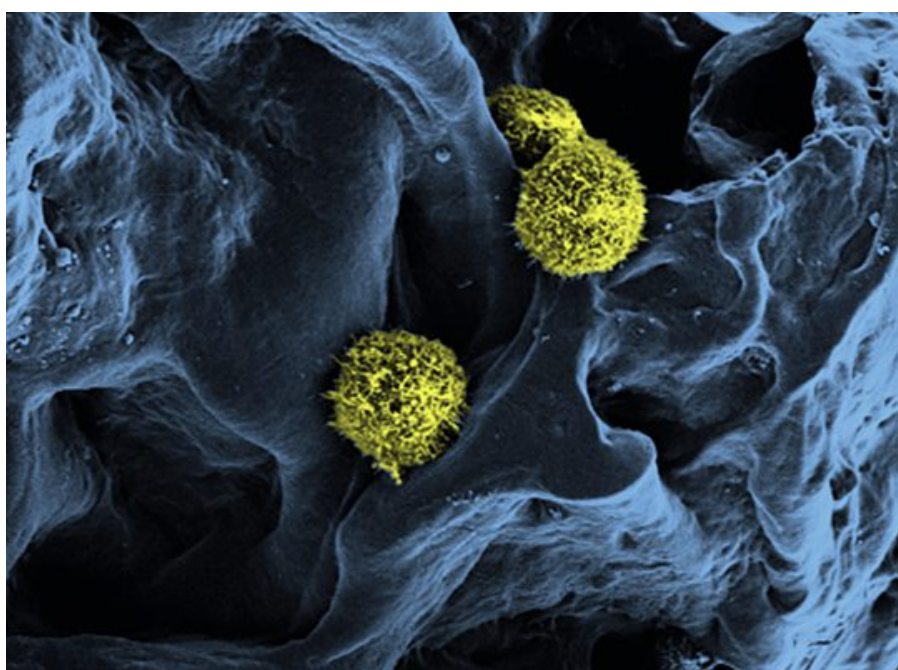

Pseudo-coloured scanning electron micrograph of hematopoietic stem cells (yellow) in a macroporous 3D hydrogel (blue). Photo: C. LeeThedieck/KIT. fluence of nanostructure, mechanical properties and architecture (2D versus $3 \mathrm{D}$ ) of the environment on HSCs. The results show that not only the biochemical composition but also the physical properties of the microenvironment influence HSC behaviour and should be considered for HSC proliferation in biomaterialbased approaches.

http://www.ifg.kit.edu/english/301.php

The research of Barbara Rothen-Rutishauser, Professor of BioNanomaterials at Adolphe Merkle Institute, Fribourg, focuses on predictive $3 \mathrm{D}$ lung models for inhalation toxicology. Investigating potential adverse effects of inhaled nanomaterials often relies on epidemiological and animal studies, but lung cell cultures in vitro are also often used. Recently, highly sophisticated 3D lung cell models have been established as they represent a more realistic physiological situation. In addition, air-liquid exposure scenarios have been developed allowing a dose-controlled deposition for materials such as spherical nanoparticles or highaspect-ratio nanomaterials, i.e. cellulose whiskers or carbon nanotubes. This approach allows scientists to fully evaluate the effect of the materials by monitoring mass deposition on the lung cell surface and to produce a dose-effect correlation.

www.am-institute.ch

Giuseppe Perale, Professor in Industrial Biotechnology at SUPSI Manno, presented ongoing research on 'New 3D Biomimetic Hydrogel to Deliver Factors Secreted by Human Mesenchymal Stem Cells in Spinal Cord Injury', carried out with his colleagues Pietro Veglianese (IRF 'Mario Negri') and Filippo Rossi (Politecnico di Milano).

Stem cell therapy with human mesenchymal stem cells (hMSCs) represents a promising strategy in spinal cord injury (SCI). However, both systemic and parenchymal hMSC administration routes show significant drawbacks, such as a limited number and viability of stem cells in situ. Biomaterials capable of encapsulating and sustaining hMSCs represent a viable approach to overcome these limitations. In their study, Giuseppe Perale and his colleagues evaluated a new agarose/carbomer-based hydrogel (HG) that combines different strategies for optimizing hMSC viability, density and delivery of paracrine factors. They were able to demonstrate that an improved combination of hMSCs and biomimetic hydrogel can significantly immunomodulate the proinflammatory environment in an SCI mouse model, increasing the M2 macrophage population and promoting a pro-regenerative



Exemplification of translational scheme of developed technology, combining hydrogels and stem cells into an implantable device. Figure: SUPSI. 
environment in situ. The scientists believe that this optimized scaffold is a promising and innovative approach, representing a possible breakthrough in using stem cell therapy supported by biomaterials with potential beneficial impact on SCI.

www.supsi.ch

To conclude the first part of the conference, which was dedicated to research, Prof. Jess Gerrit Snedeker from University of Zurich, Department of Orthopaedics and Deputy Head of the Institute for Biomechanics at the ETH Zurich explained recent studies on Multi-scale Functional Imaging of Tendon and its progression from Bench to Bedside. Prof. Snedeker introduced 'collagen ageing' as a potential driver of connective tissue disease. The lecture focused specifically on experimental imaging tools and techniques for untangling tendon structure/function relationships across many metric orders of magnitude, from the collagen molecule to the patient. At the molecular level, the focus is on non-enzymatic reaction of proteins with glucose (glycation) as a topic of rapidly growing importance in human health and medicine. There is increasing evidence that this reaction plays a central role in ageing and connective tissue diseases. Of particular interest are glycation-related changes in type-I collagens, the long-lived proteins that form the mechanical backbone of musculoskeletal connective tissues such as tendons and tissues in almost every other human organ. Despite considerable correlative evidence relating extracellular matrix (ECM) glycation to disease, little is known about how ECM modification by glucose impacts matrix mechanics and damage, cell-matrix interactions, and matrix turnover during ageing. More daunting is the challenge to understand how these factors interact so as to cumulatively affect local repair of matrix damage, progression of tissue disease or systemic health and longevity. The lecture summarized what is currently known regarding collagen glycation as a potential driver of connective tissue disease, and how tendon damage and repair can be quantified using imaging techniques in the laboratory and clinic.

www.biomech.ethz.ch

Opening the second part of the event, which focused on industrial applications, Dr. Christian N. Parker from the Novartis Institute for BioMedical Research reported on 'Simple Assays for Complex Biology'. His presentation described a simple assay that monitors the metabolic activity of intestinal-derived crypts. These crypts then serve as an ex vivo model of radiation-induced intestinal mucositis. The assay uses mouse-derived primary intestinal crypts grown in a complex 3D matrix. Control treatments were shown to promote crypt recovery after irradiation, as occurs in vivo. The assay was then used to screen a focused set of compounds, with known targets, allowing the identification of compounds that increased recovery from irradiation. The tool compounds revealed subtle changes in crypt growth and their response to external stimuli, allowing further characterization of the system. It is hoped that such ex vivo, 3D, physiologically rel-

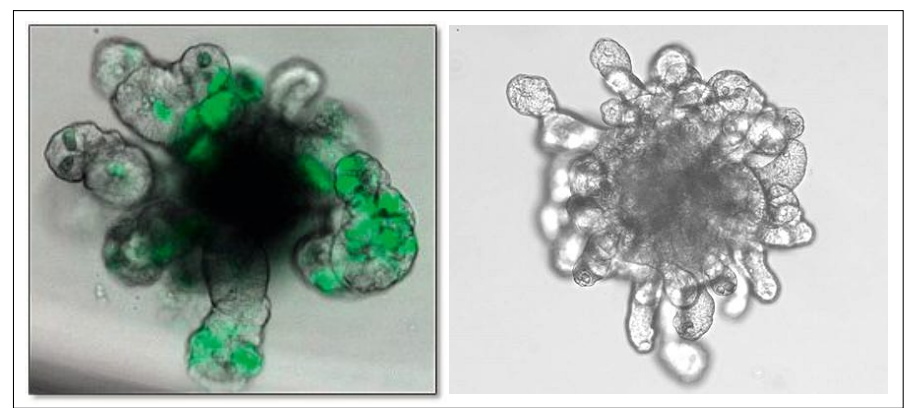

Microscope image of an intestinal crypt growing in Matrigel and expressing a GFP protein linked to the Lgr5 promoter. Photo: Novartis. evant, organoid systems can serve as a bridge between relatively simple in vitro assays using 2D immortalized cell lines and complex in vivo models using whole animals.

https://www.nibr.com/

\section{State-of-the-Art Implementations in Industry}

The second group of short talks was given by industry representatives of TEDD members, starting with Dr. Isabel Patocchi, Application Specialist at Tecan Schweiz AG. She presented the 'Parallel Preparation of RAFT 3D Cell Cultures on the Freedom EVO $^{\circledR}$ '. TAP Biosystems has developed a 3D cell culture technique - RAFT (Real Architecture for 3D Tissue) - that allows researchers to culture their choice of cell type, including primary cells, in an in vivo-like environment. RAFT is now distributed exclusively by Lonza BioSciences. The automated parallel preparation of four RAFT 3D cell cultures on the Freedom EVO workstation offers the potential to produce eight 96-well plates of RAFT 3D cell cultures per day or 40 plates (almost 4,000 single wells) per working week. With this increased throughput, experiments can be carried out in parallel, making results available in a shorter timeframe. Culturing of the HCT-116 cancer cell line in a 3D environment facilitates the formation of the typical tumoroid structures and viability assays and enables dose-response studies to be performed easily and reproducibly in a realistic environment.

www.tecan.com

Dr. Elise Demange is Chairperson and CSO at CELENYS, an innovative biotechnology company in France. CELENYS develops scaffolds for $3 \mathrm{D}$ cell culture to better mimic the tissue microenvironment and provide more predictive in vitro tools for drug development. She focused on 'Hyaluronic Acid Scaffolds for 3D Cell Culture', especially Biomimesys ${ }^{\circledR}$, a hyaluronan scaffold that overcomes the 2D flat culture limitations and mimics the in vivo physiology within the in vitro environment. It facilitates cell-matrix interaction and cell-cell interaction and recreates a more physiologically authentic 3D architecture of tissues and organs for advanced drug discovery, ADME-Tox profiling, disease modelling. Biomimesys ${ }^{\circledR}$ consists of HA chains with the crosslinking agent $\mathrm{ADH}$ (adipic dihydrazide acid). Elise Demange and her team studied the reorganization of cellular cytoskeleton in $3 \mathrm{D}$ cell culture and the viability and development of multicellular spheroids within the hydrogel. As she reported, Biomimesys ${ }^{\circledR}$ scaffold technology is physiological, easy to use and compatible with all standard analysis methods. Data characterizing the formation of hyaluronan scaffold spheroids are available and highlight the cell morphology and proliferation rate. Biomimesys ${ }^{\circledR}$ has also been used successfully with various cell types including cancer cell lines, human primary cells and stem cells. These results highlight the use of Biomimesys ${ }^{\circledR}$ to grow 3D cell culture as a relevant model for drug screening.

www.celenys.com

The series of short talks was closed by Dr. Remo Campiche, Study Director at DSM Nutritional Products, one of the world's leading suppliers of vitamins, carotenoids and other ingredients to the feed, food, pharmaceutical and personal care industries. His concern is 'A Claim Substantiation for Resveratrol as an Inhibitor of Skin Pigmentation using Reconstructed Human Pigmented Epidermis'. Skin lightening and an even skin tone are important topics in the cosmetic field. While, in the Asian population, fair skin plays an important role in people's beauty aspirations, in Western societies people are more concerned with an even skin tone and the fight against age spots. Remo Campiche and his colleagues believe that the quest for perfect skin is a powerful driver for scientific research. Therefore, their goal is to 
obtain a comprehensive and scientifically sound claim substantiation for the development of cosmetic actives. In line with this objective, he is generating an exemplary multi-parameter dataset for resveratrol as an inhibitor of skin pigmentation. At DSM, they use 3D tissue culture techniques with human melanocytes and human keratinocytes. Pigmentation was assessed using melanin extraction to quantitate melanin content and chromameter measurements (luminosity) in addition to visual assessment. For mechanistic studies, DSM monitored key melanogenesis markers by immunochemical analysis of 3D-tissue culture sections. Using 3D-tissue culture they showed that various parameters like melanin content, luminosity, or visual pigmentation are reduced by resveratrol. In order to shed light on the mechanism behind melanogenesis inhibition, the scientists histologically showed the down-regulation of markers associated with melanogenesis, such as tyrosinase or Melan-A. Overall, they obtained a comprehensive picture of the efficacy of resveratrol as an inhibitor of cutaneous pigmentation. In addition, they were able to show that 3D-tissue culture is a valuable tool for scientific claim substantiation related to cosmetic active ingredients.

www.dsm.com

One of the highlights of the meeting was the contribution of

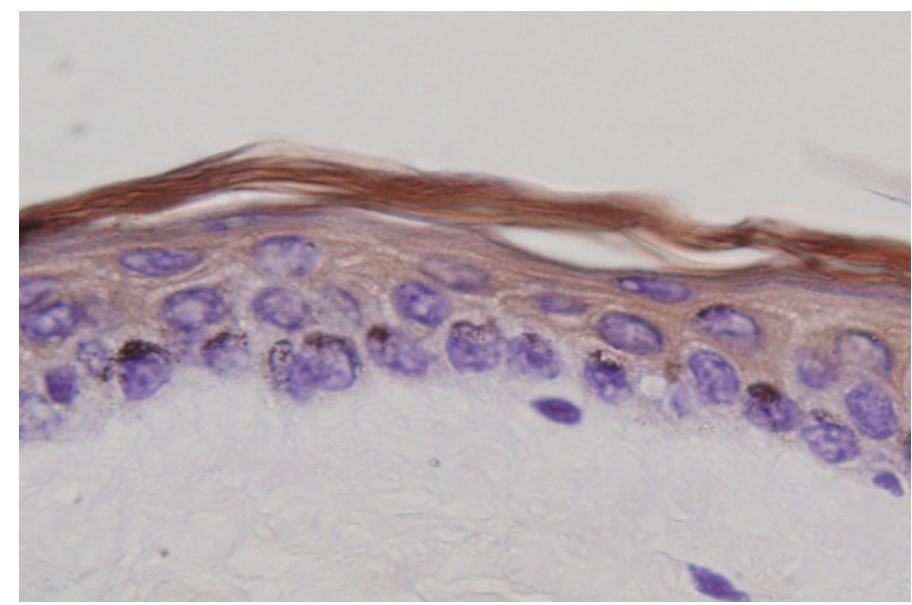

Skin section with brown melanin granules giving the skin its pigmentation. This forms the basis for Remo Campiche and his team to show, using 3D-tissue culture, that various parameters like melanin content, luminosity, or visual pigmentation are reduced by resveratrol. Photo: Remo Campiche.
Dr. Jos Joore, co-founder and managing director of Mimetas B.V., Leiden, the Netherlands, with his talk about the 'Revolutionizing Organ-on-a-chip Technology: Any throughput, any complexity, anywhere'. MIMETAS develops organ-on-a-chip tissue models for evaluating drugs, chemicals and food components. The company's unique microfluidic technology, designated OrganoPlates ${ }^{\mathrm{TM}}$, enables compounds to be tested with high-throughput on miniaturized human organ models, replacing laboratory animals and conventional cell culture models. The OrganoPlate ${ }^{\mathrm{TM}}$ platform supports $3 \mathrm{D}$ cell culture under continuous perfusion and membrane-free co-culture, including epithelial and endothelial boundary tissues. MIMETAS develops and validates customized disease, toxicology and transport models and will ultimately make its technology available for personalized therapy selection.

www.mimetas.com

The TEDD Annual Meeting 2015 was a success. One highlight of the well-attended conference was the exhibition in the exotic atmosphere of the greenhouse at ZHAW where representatives from academia and industry provided a glimpse at their activities.

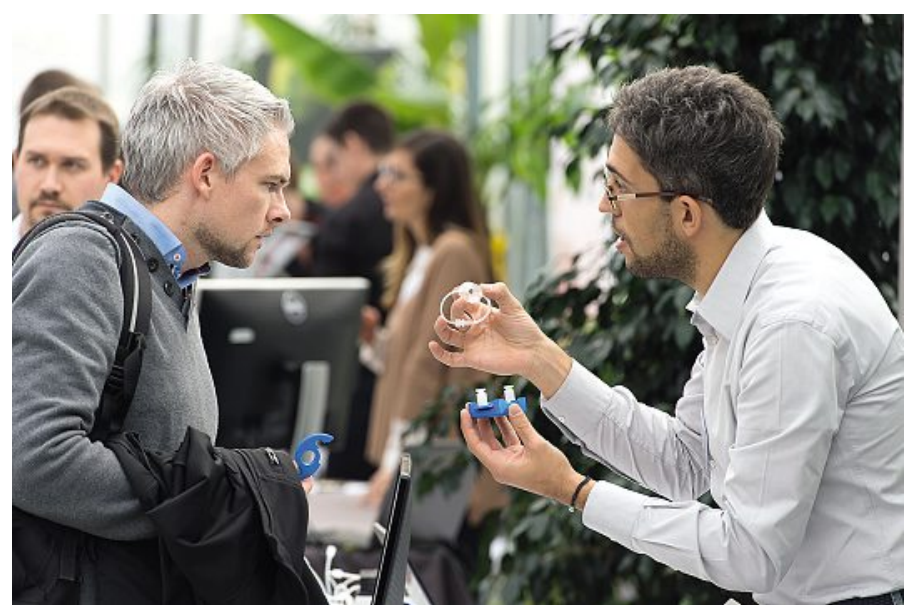

As one of the 15 exhibitors, the company IVTech srl from Italy demonstrated their devices for 3D cell culture.

At the end of the conference, which marks the $5^{\text {th }}$ anniversary of the TEDD network, Ursula Graf-Hausner draws a positive conclusion on the whole: "Over the last five years we have built up a strong and successful network, Now we are ready to address the implementation of our 3D in vitro models for routine application in research and industry."

www.icbc.zhaw.ch/tedd 